\title{
Calidad de vida laboral en docentes chilenos
}

\section{Quality of working life in Chilean teachers}

\author{
Andrea Victoria Miño Sepúlveda* \\ Universidad de Santiago de Chile \\ (Rec.: agosto de 2015 - Acept.: septiembre de 2016)
}

\begin{abstract}
Resumen
Este artículo aborda la temática de la calidad de vida laboral de los profesores de Enseñanza Básica y Enseñanza Media en Chile. Para ello, se describen y analizan los resultados obtenidos por medio del Cuestionario CVP-35 (Karasek, 1989) en una muestra de 380 docentes del territorio nacional. La finalidad de la investigación descrita en este artículo es caracterizar a la muestra de docentes chilenos respecto de su nivel de calidad de vida laboral, analizando los indicadores que dan cuenta de la realidad del trabajo que desempeñan y efectuando comparaciones entre variables socio-demográficas. Los resultados indican que en cuanto a la percepción de apoyo directivo y su motivación intrínseca, la muestra de docentes los califica como adecuados. Sin embargo, da cuenta de una alta carga de trabajo, manifestada en falta de tiempo para la vida personal, cantidad de trabajo, estrés, prisas y agobios, carga de responsabilidad, baja satisfacción con el sueldo y dificultad para desconectarse al acabar la jornada de trabajo.
\end{abstract}

Palabras clave: calidad de vida laboral, profesores, administración de recursos humanos.

\begin{abstract}
This article discusses the quality of working life of elemental and secondary teachers in Chile. The results are described and analyzed by the CVP-35 (Karasek, 1989) in a sample of 380 Chilean teachers. The objective was to characterize a sample of Chilean teachers about the quality of their working life, analyzing indicators related with those conditions under which they performed their work and making comparisons between socio-demographic variables. The results pointed out both the perception of management support and the intrinsic motivation of teachers in the sample as suitable. However, they account for a high workload manifested in lack of time for personal life; amount of work; stress; hustle and hassles; burden of responsibility; low satisfaction with salary and difficulty to disconnect at the end of the workday.
\end{abstract}

Keywords: quality of working life, teachers, human resources management.

\footnotetext{
* Correspondencia a: Andrea Miño Sepúlveda. Avenida Pedro de Valdivia 0193. Providencia. Santiago. E-mail: andrea.mino@usach.cl
} 


\section{Introducción}

En el ámbito de las instituciones educacionales, Weinstein (2002) indica que hoy en día es necesario que predomine una visión amplia de las organizaciones más que una mirada administrativa y formal. Se debe considerar la gestión de los recursos físicos, de las personas, de los procesos, del entorno y de los resultados; todos ellos son elementos que interactúan recíprocamente dentro de cualquier organización. Se debe considerar una gestión institucional racional, en el sentido que la coordinación de los esfuerzos se refleje en acciones cooperativas que se orienten al logro de objetivos compartidos, cuyos resultados sean evaluados para generar una retroalimentación a las acciones emprendidas para una gestión eficaz (Villarreal, 2005). Los avances en el área de la gestión junto a las teorías sistémicas de las organizaciones y los modelos de calidad y de excelencia han contribuido a desplazar la mirada sobre la gestión desde una visión administrativa y formal de la organización hacia una preocupación por el conjunto de factores y procesos que inciden en la gestión de ella y el logro de sus resultados.

En el contexto de las organizaciones ha persistido la frase de que "nuestra gente es nuestro activo más importante", pero cabe la interrogante si esto es un estereotipo, dado que pareciera ser que son pocas las organizaciones educativas que respetan este principio en la práctica cotidiana. De hecho, Arellano (2005) indica que uno de los actores principales es la persona del docente, pero que estos manifiestan inseguridad respecto de las materias que imparten, derivado de la oferta dispar en cuanto a perfeccionamiento docente y la calidad de los programas impartidos. En el avance hacia una relación más bien de socio estratégico de la organización, recursos humanos debe concentrarse en el exterior, comparando su institución con las otras organizaciones y recopilando y analizando datos acerca de cómo son las políticas y estrategias de gestión del talento necesarias para cumplir con sus objetivos de manera eficiente. Para efectuar este diagnóstico, se hace necesario que los Directores amplíen su mirada, siendo necesario que se mantengan informados sobre los avances en el área y de las mejores prácticas que se están llevando a efectos en las diversas organizaciones del medio. Asimismo, debe poseer un bagaje de conocimientos actualizado para responder de manera fundamentada a las exigencias que le presenta el medio.

Respecto de lo anterior, se tiene evidencia de estudios realizados en el ámbito de la dirección de las organizaciones educativas, en que autores como Genesi \& Suárez (2010) dejan al descubierto variables propias de la gestión dentro de los centros educativos que se relacionan con la satisfacción que alcanzan los profesores. De esta manera, factores como la participación, contribución, responsabilidad y liderazgo son claves para un desempeño efectivo del cuerpo docente y que se correlacionarían con la calidad de vida laboral (en adelante $\mathrm{CVL}$ ) de estos profesionales. En lo relativo al liderazgo y eficacia colectiva, se ha encontrado evidencia que los relaciona como variables mediadoras en la satisfacción laboral en los docentes (López \& Gallegos, 2014). Es por ello que este estudio busca diagnosticar la CVL en una muestra de docentes de enseñanza básica y enseñanza media chilenos y proporcionar una panorámica de qué elementos influencian y se relacionan con una adecuada calidad de vida docente y qué elementos pudiesen ser considerados protectores en relación con aquella.

En términos generales, los cambios demográficos, sociales y culturales que se han sucedido a lo largo de las últimas décadas han generado una dificultad en las personas para encontrar un equilibrio entre su vida personal y su trabajo (Cornejo, 2009; Romeo, Yepes-Baldó \& Berger, 2014). Desde otra perspectiva, se han contrapuesto los cambios en el mercado laboral y el aumento del empleo de las mujeres, con el deseo de lograr mayores niveles de calidad de vida a través de actividades de tiempo libre y de ocio (Bee, Baskar \& Vimala, 2013; Cegarra-Leiva, Sánchez-Vidal \& Cegarra-Navarro, 2012; Maxfield, 2005; Ollier-Malaterre, Valcour, Den Dulk \& Kossek, 2013).

Respecto de Chile, coexiste el progreso económico y social con una menor percepción de calidad de vida laboral. Esto se refleja en estadísticas que consideran que Chile es uno de los países líderes en Latinoamérica en cuanto a crecimiento y estabilidad de su economía (América Economía, 2016). Por otra parte, cuenta con el status de líder latinoamericano en lo referido a desarrollo humano (Programa de las Naciones Unidas para el Desarrollo [PNUD], 2012, 2014) con las menores cifras en pobreza, mayor cobertura de educación, mejores niveles de infraestructura, seguridad y de cobertura de servicios básicos. De este modo, entre los años 1980 y 2013 la esperanza de vida en Chile se incrementó en 10 años, así como también los años de escolaridad en los adultos aumentaron en 3 años. La otra cara de la moneda se pone en evidencia con indicadores que sitúan a Chile como uno de los países de la OCDE en donde más horas se trabaja, con una media de 1990 horas de empleo versus 1770 horas de trabajo de los países pertenecientes a la OCDE (ENCLA, 2015). Por otra parte, se tienen los índices de estrés más altos de la región, con altas tasas de depresión, insatisfacción laboral y problemas de salud laboral (Dirección del Trabajo, 2011; Garrido et al., 2013; Guic, Bilbao \& Bertin, 2002; Romanik, 2011).

\section{Calidad de Vida}

En términos generales, el concepto calidad de vida surge como objeto de interés académico, tras el pe- 
ríodo de la Segunda Guerra Mundial, a partir de la percepción de que el crecimiento económico no solo debe considerar un incremento en el consumo, crecimiento de la riqueza y desarrollo de los mercados, sino que, además, debe ser un medio que permita a las personas alcanzar mejores condiciones de vida (Rueda, 1996; Urzúa \& Caqueo- Urizar, 2012; Verdugo, Guzmán, Moy, Meda \& González, 2008).

Por otra parte, el concepto de CVL tiene sus orígenes hacia fines de los años 60 y comienzos de los 70, gracias a una serie de conferencias realizadas por el Ministerio de Trabajo de los Estados Unidos de América y la Fundación Ford, en donde French (como se citó en González, Hidalgo \& Salazar, 2006) aludió a que calidad de vida es una filosofía que busca una mejora de las condiciones del trabajador, efectuando cambios culturales que propendan a su desarrollo y progreso personal. Desde esta definición, se concluyó que la CVL era un concepto que no sólo se refería a la satisfacción en el trabajo, sino que también incluía otras dimensiones como la participación en la toma de decisiones, autonomía en la gestión, puestos de trabajos, sistemas y estructuras organizacionales que propenden al bienestar físico y psicológico de los sujetos.

Desde entonces y no sin problemas, los investigadores han tratado de alcanzar un consenso respecto del significado y el alcance del concepto calidad de vida, encontrándose como una definición aclaratoria y generalmente compartida la de "calidad de la vivencia que de la vida tienen los sujetos" (Rueda, 1996, p. 30). Se comprende que la calidad de vida es un concepto multidimensional que contiene elementos de bienestar, de políticas sociales, dimensiones materiales y no materiales, objetivas y subjetivas, individuales y colectivas (Nava, 2012); en donde se consideran variables tales como horario de trabajo, remuneración, recompensas, beneficios, clima organizacional, oportunidades de desarrollo mediante planes de carrera y relaciones interpersonales, entre otras (Casas, Lorenzo \& Cañas, 2002).

En la misma senda, Walton (1973) mencionó ocho dimensiones respecto de $\mathrm{CVL}$, siendo estas: trabajo y espacio total de vida, integración social de la vida en el trabajo, oportunidad de desarrollo, compensación justa y adecuada, reglamentación, condiciones de seguridad y salud en el trabajo, importancia social de la vida en el trabajo, y oportunidad de crecimiento continuo.

Dada la naturaleza dinámica del concepto, Segurado y Agulló (2002) indican la existencia de dos dimensiones dentro del estudio de la CVL, aludiendo a una perspectiva que se centra en las condiciones del entorno de trabajo y que implica un nivel de análisis macro, con foco en el logro de los objetivos organizacionales. En segundo lugar, se encuentra la dimensión psicológica, la cual se centra en el bienestar de la persona, inclu- yendo las vivencias del trabajador, situándolo como el eje primordial de análisis.

Si bien en Chile las aproximaciones al estudio de la calidad de vida laboral han sido desde la perspectiva médica y de la salud ocupacional, durante los últimos años la CVL pareciera haberse convertido en un tema que concita cada vez más interés por parte de las empresas en el país. Este hecho se encuentra aparentemente gatillado por las consecuencias directas e indirectas del ritmo de vida actual, que finalmente está afectando negativamente los resultados organizacionales a través de la baja productividad y la mala calidad del trabajo diario (Cañón \& Martínez, 2011; Romeo et al., 2014; Segurado \& Agulló, 2002).

Llevando el concepto de CVL al ámbito docente, se encuentra que los profesores dan cuenta de condiciones adversas que deben enfrentar en la realización de sus funciones, las cuales afectarían a su salud física y psicológica. Entre estas se destacan las condiciones relacionadas con la disponibilidad de recursos e infraestructura, las condiciones socioculturales y ambientales que rodean el establecimiento educacional, las condiciones de higiene y seguridad en el trabajo y las regulaciones percibidas como excesivas que provienen del nivel central y local, con un porcentaje de horas lectivas en aula que supera el máximo legal permitido. Esto da paso a una gran cantidad de horas extra dedicadas a tareas docentes, lo cual se suma a la inexistencia de minutos de descanso durante la jornada laboral (Guerrero \& Rubio, 2008; Parra, 2005; Redondo, 2009).

Las condiciones anteriormente descritas han llevado a un creciente malestar entre los docentes tanto de enseñanza básica como de enseñanza media, encontrándose por ejemplo que en España, un 50\% de estos profesionales manifiestan ansiedad, un $16 \%$ muestran indicadores de depresión y un $10 \%$ de los profesores han optado por retirarse de sus funciones (Sánchez, March y Cerda \& Ballester, 2015). Asimismo, Cornejo (2009) señala que en una muestra de docentes de enseñanza secundaria de Santiago, se encuentra que un $13,9 \%$ reporta haber sufrido de estrés y un $13,2 \%$ indica haber padecido depresión durante los últimos dos años.

\section{Métodología}

\section{Diseño de Investigación}

El presente estudio se desarrolló mediante un método de corte cuantitativo, con un tipo de investigación descriptivo y un diseño de estudio de carácter no experimental (Hernández, Fernández \& Baptista, 2010). El tipo de diseño no experimental que se utilizó es el diseño transeccional descriptivo, ya que se recolectaron datos en un solo momento, en un tiempo único, con el propósito de describir las variables en un mo- 
mento dado.

\section{Muestra}

La población a la cual estuvo dirigido el estudio fue la de profesores chilenos. De ésta, se seleccionó una muestra de 380 docentes que ejercieran en Enseñanza Básica y Enseñanza Media en establecimientos educacionales municipales, particulares subvencionados y particulares pagados del territorio nacional. El diseño muestral se centró en una muestra no probabilística de sujetos voluntarios (Hernández et al., 2010), la cual se refiere a un tipo de muestreo por conveniencia, por lo que como Zinser (1987) señaló, los sujetos participantes en el estudio corresponden a aquellos que estuvieran más disponibles para los investigadores. Se escogió este tipo de muestreo de modo de hacer más flexible la selección de los sujetos participantes en la investigación y por la facilidad y rapidez del método. Este permite aceptar a todo aquel sujeto que, cumpliendo con las características señaladas, acepta ser parte del estudio. Por otra parte, cabe señalar que se consideró tanto a profesores de enseñanza básica como de enseñanza media, dado que si bien cada uno de ellos presenta particularidades respecto de los alumnos que atiende, se optó por la visión macro para el estudio, al igual que al considerar la aplicación del cuestionario a los distintos tipos de dependencia de los establecimientos en los cuales se desempeñan.

\section{Instrumento}

Se empleó el Cuestionario CVP-35 (Karasek, 1989) validado y adaptado por Cabezas (1998). Se trata de un instrumento autoadministrado que efectúa una medida multidimensional de la calidad de vida profesional, evaluando la experiencia de bienestar derivada del equilibrio que percibe el individuo entre las demandas de trabajo y los recursos psicológicos, organizacionales y relacionales de que dispone para afrontarlas. Consta de 35 preguntas que se responden en una escala del 1 al 10, con categorías que van desde «nada» (valores 1 y 2), «algo» (valores 3, 4 y 5), «bastante» (valores 6, 7 y 8) y «mucho» (valores 9 y 10). El cuestionario cuenta con 3 factores «apoyo directivo», "cargas de trabajo» y «motivación intrínseca». Caboblanco et al. (2004) evaluaron las características métricas de este instrumento en personal de salud y encontraron una consistencia interna aceptable para los factores $(0,70)$ y alta para la puntuación global $(0,81)$.

\section{Resultados}

La Tabla 1 muestra la distribución de las variables socio-demográficas de la muestra de docentes participantes en esta investigación. La muestra se encontraba constituida en un $81,1 \%$ por profesoras. La edad de los participantes en su mayoría correspondía al rango de 26 a 30 años. Además, un 59,7\% de los profesores se encontraba soltero. En cuanto a experiencia laboral
, un $35,8 \%$ de los participantes se hallaba en la etapa de Diversificación- Cuestionamiento (7 a 25 años de experiencia laboral), en la cual la persona adopta una actitud de cambio de sus metodologías y formas de evaluación, junto con un replanteamiento de su futuro en la docencia (Ponce, 2002). En términos de permanencia en el establecimiento educacional actual, los profesores de la muestra habían trabajado mayoritariamente un período de 1 a 5 años en el mismo lugar. Del total de la muestra, un $64,2 \%$ se desempeñaba en enseñanza básica y un 61,6\% trabajaba en establecimientos de dependencia particular subvencionada. Finalmente, respecto a la región en la cual ejercían sus funciones, un $62,1 \%$ de los participantes lo hacía dentro de la Región Metropolitana, seguido de un 8,7\% que se desempeñaba en la $\mathrm{V}$ Región de Valparaíso y un $8,2 \%$ que ejercían su labor docente en la VIII Región de Bío Bío.

En la Tabla 2 se encuentran las puntuaciones que otorgó la muestra de docentes participantes en el estudio a los tres factores del instrumento y su puntuación total para el ítem Calidad de vida. Como apuntamos anteriormente, la categorización que se estableció para las respuestas discurre entre "nada" atribuida a valores entre 1 y 2 ; "algo" (valores entre 3 y 5); "bastante" (valores entre 6 y 8) y "mucho" (valores entre 9 y 10). De este modo, para el Factor I: Apoyo directivo, la muestra de docentes otorgó un puntaje promedio de 6,47 correspondiendo a la categoría de "bastante". En cuanto al Factor II: Carga de trabajo, se aprecia que la muestra de profesores lo puntuó con un promedio de 6,64, siendo categorizado como "bastante". El Factor III: Motivación intrínseca, contó con una puntuación promedio de 5,17, considerándose igualmente dentro de la categoría "bastante". Finalmente, el promedio del total de las puntuaciones de los profesores en la prueba fue de un 6,14. Esto significa que, en general, tuvieron una adecuada percepción de calidad de vida profesional.

Como se aprecia en la Tabla 3, los docentes concedieron altas puntuaciones a ítems como: Falta de tiempo para la vida personal; Cantidad de trabajo; Estrés; Prisas y agobios; Carga de responsabilidad. Por el contrario, los docentes entregaron puntuaciones bajas a los ítems Satisfacción con el sueldo y Me desconecto al acabar la jornada de trabajo.

La Tabla 4 muestra las puntuaciones concedidas por los docentes a cada uno de los factores del Cuestionario de Calidad de Vida, categorizándose este análisis en función de la variable Género. Así se encontraron diferencias estadísticamente significativas entre mujeres y hombres solo para el Factor III: Motivación intrínseca, siendo los profesores quienes otorgaron una mayor puntuación a este factor (RP: 220,17; Sig. 0,011).

En la Tabla 5 se da cuenta de la existencia de diferencias significativas para el Factor I: Apoyo directivo y 
Tabla 1. Distribución variables socio-demográficas en la muestra de docentes

\begin{tabular}{|c|c|c|c|c|}
\hline Variables Socio-dem & lográficas & $\begin{array}{l}\text { Frecuen- } \\
\text { cia }\end{array}$ & $\begin{array}{l}\text { Porcen- } \\
\text { taje }\end{array}$ & $\begin{array}{l}\text { Porcentaje } \\
\text { Acumulado }\end{array}$ \\
\hline Género & $\begin{array}{l}\text { Femenino } \\
\text { Masculino }\end{array}$ & $\begin{array}{c}308 \\
72\end{array}$ & $\begin{array}{r}81,1 \\
18,9\end{array}$ & $\begin{array}{l}81,1 \\
100\end{array}$ \\
\hline Edad & $\begin{array}{l}22 \text { a } 25 \text { años } \\
26 \text { a } 30 \text { años } \\
31 \text { a } 35 \text { años } \\
36 \text { a } 40 \text { años } \\
41 \text { a } 45 \text { años } \\
46 \text { a } 50 \text { años } \\
51 \text { a } 55 \text { años } \\
56 \text { a } 60 \text { años } \\
61 \text { a } 65 \text { años }\end{array}$ & $\begin{array}{c}55 \\
142 \\
81 \\
41 \\
15 \\
19 \\
17 \\
9 \\
1\end{array}$ & $\begin{array}{c}14,5 \\
37,4 \\
21,3 \\
10,8 \\
3,9 \\
5,0 \\
4,5 \\
2,4 \\
0,2\end{array}$ & $\begin{array}{l}14,5 \\
51,9 \\
73,2 \\
84,0 \\
87,9 \\
92,9 \\
97,4 \\
99,8 \\
100\end{array}$ \\
\hline $\begin{array}{l}\text { Años de Experiencia } \\
\text { Laboral }\end{array}$ & $\begin{array}{l}\text { Inicio de Carrera ( } 1 \text { a } 3 \text { años) } \\
\text { Estabilización ( } 4 \text { a } 6 \text { años) } \\
\text { Diversificación- Cuestionamiento ( } 7 \text { a } 25 \text { años) } \\
\text { Serenidad- Conservadurismo ( } 25 \text { a } 35 \text { años) } \\
\text { Preparación a la Jubilación ( } 35 \text { a } 40 \text { años) }\end{array}$ & $\begin{array}{c}127 \\
95 \\
136 \\
20 \\
2\end{array}$ & $\begin{array}{c}33,4 \\
25,0 \\
35,8 \\
5,3 \\
0,5\end{array}$ & $\begin{array}{l}33,4 \\
58,4 \\
94,2 \\
99,5 \\
100\end{array}$ \\
\hline $\begin{array}{l}\text { Años de Permanen- } \\
\text { cia en el Estableci- } \\
\text { miento }\end{array}$ & $\begin{array}{l}1 \text { a } 5 \text { años } \\
6 \text { a } 10 \text { años } \\
11 \text { a } 15 \text { años } \\
16 \text { a } 20 \text { años } \\
21 \text { a } 25 \text { años } \\
26 \text { a } 30 \text { años } \\
31 \text { a } 35 \text { años }\end{array}$ & $\begin{array}{c}313 \\
32 \\
15 \\
9 \\
4 \\
6 \\
1\end{array}$ & $\begin{array}{c}82,4 \\
8,4 \\
3,9 \\
2,4 \\
1,1 \\
1,6 \\
0,3\end{array}$ & $\begin{array}{c}82,4 \\
90,8 \\
94,7 \\
97,1 \\
98,2 \\
99,7 \\
100 \\
\end{array}$ \\
\hline Nivel Educacional & $\begin{array}{l}\text { Enseñanza Básica } \\
\text { Enseñanza Media }\end{array}$ & $\begin{array}{l}244 \\
136 \\
\end{array}$ & $\begin{array}{l}64,2 \\
35,8\end{array}$ & $\begin{array}{l}64,2 \\
100\end{array}$ \\
\hline Región & $\begin{array}{l}\text { I Región de Tarapacá } \\
\text { II Región de Antofagasta } \\
\text { III Región de Atacama } \\
\text { IV Región de Coquimbo } \\
\text { V Región de Valparaíso } \\
\text { VI Región de O'Higgins } \\
\text { VII Región de Maule } \\
\text { VIII Región de Bío Bío } \\
\text { IX Región de La Araucanía } \\
\text { X Región de Los Lagos } \\
\text { XI Región de Aysén } \\
\text { XII Región de Magallanes y la Antártica Chilena } \\
\text { RM Región Metropolitana } \\
\text { XIV Región de Los Ríos } \\
\text { XV Región de Arica y Parinacota }\end{array}$ & $\begin{array}{c}0 \\
3 \\
1 \\
16 \\
33 \\
20 \\
10 \\
31 \\
11 \\
13 \\
1 \\
1 \\
236 \\
4 \\
0\end{array}$ & $\begin{array}{c}0,0 \\
0,8 \\
0,3 \\
4,2 \\
8,7 \\
5,3 \\
2,6 \\
8,2 \\
2,9 \\
3,4 \\
0,3 \\
0,3 \\
62,1 \\
1,1 \\
0,0\end{array}$ & $\begin{array}{c}0,0 \\
0,8 \\
1,1 \\
5,3 \\
13,9 \\
19,2 \\
21,8 \\
30,0 \\
32,9 \\
36,3 \\
36,6 \\
36,8 \\
98,9 \\
100 \\
100\end{array}$ \\
\hline Estado Civil & $\begin{array}{l}\text { Soltero/a } \\
\text { Casado/a } \\
\text { Viudo/a } \\
\text { Separado/a } \\
\text { Divorciado/a }\end{array}$ & $\begin{array}{c}227 \\
111 \\
2 \\
28 \\
12\end{array}$ & $\begin{array}{c}59,7 \\
29,2 \\
0,5 \\
7,4 \\
3,2\end{array}$ & $\begin{array}{l}59,7 \\
88,9 \\
89,5 \\
96,8 \\
100\end{array}$ \\
\hline
\end{tabular}


para Factor II: Carga de trabajo, en función de la variable Tipo de dependencia. Los docentes que se desempeñan en el sector municipal contaban con una percepción de mayor apoyo directivo en sus establecimientos para realizar sus funciones. Además, asignaron mayores puntuaciones al factor Carga de trabajo en comparación con los docentes de los sectores particulares subvencionados y particulares pagados.

En la Tabla 6 se encuentran diferencias estadísticamente significativas entre los docentes que desempeñan su función en la Región Metropolitana y quienes trabajan en regiones. Los docentes de la Región Metropolitana dieron cuenta de una mayor carga de trabajo, mayores índices de motivación intrínseca y, en general, una mejor calidad de vida laboral en comparación con sus colegas de regiones.

\section{Discusión}

El término calidad de vida laboral alude a un concepto multidimensional compuesto por una dimensión material (referida a las condiciones en las cuales se desempeña el trabajo) y, por otra parte, a aquella dimensión
Maureira (2006) presenta una serie de ejemplos sobre las prácticas directivas en los establecimientos educaciones de tres países sudamericanos. Así, en Argentina, la eficacia se encuentra en la cultura y la gestión escolar ligada a un liderazgo a través del cual sus integrantes construyen visiones compartidas y orientaciones estratégicas. Por otra parte, en Bolivia, la gestión escolar se encuentra asociada a una definición clara de los objetivos institucionales y del ambiente escolar. En Brasil, resaltan el rol del liderazgo del director del establecimiento como factor de eficacia en la gestión. Finalmente, en Chile, se ubican como importantes el sentido de misión, las normas, el clima organizacional, la estructura organizacional y el liderazgo del director expresado en su competencia para dar orientación al proceso y ejercer supervisión.

Dado que se han hallado altas puntuaciones en la percepción de apoyo del director del establecimiento educativo y de su equipo directivo, se debe hacer hincapié en que son ellos los principales involucrados en promover el trabajo en equipo, proporcionar feedback

Tabla 2. Distribución de puntuaciones en cada dimensión

\begin{tabular}{lllll}
\hline & $\begin{array}{l}\text { Factor I: Apoyo direc- } \\
\text { tivo }\end{array}$ & $\begin{array}{l}\text { Factor II: Carga de tra- } \\
\text { bajo }\end{array}$ & $\begin{array}{l}\text { Factor III: Motivación } \\
\text { intrínseca }\end{array}$ & $\begin{array}{l}\text { Calidad de vida profe- } \\
\text { sional }\end{array}$ \\
\hline $\mathrm{n}$ & 380 & 380 & 380 & 380 \\
Media & 6,47 & 6,64 & 5,17 & 6,14 \\
Desv. típ. & 1,155 & 1,245 & 1,461 & 1,108 \\
\hline
\end{tabular}

del bienestar subjetivo. En el desarrollo de esta investigación se fue indagando sobre la percepción de calidad de vida laboral en una muestra de 380 docentes que ejercen sus labores en Enseñanza Básica y Enseñanza Media en el territorio nacional.

En primer lugar, los resultados de la investigación dan cuenta de un perfil respecto de la calidad de vida laboral de los docentes chilenos, encontrándose que en la actualidad, los profesores manifiestan, en promedio, una adecuada calidad de vida laboral, expresándose en motivación intrínseca en el cuerpo docente y una positiva percepción de apoyo de parte de la dirección del establecimiento. Estos factores se constituyen en protectores respecto del malestar en el profesorado, incrementando la percepción de CVL, por lo cual sería de suma importancia potenciar estos elementos al interior de los establecimientos educacionales. Al respecto Goswami (2013) indicó que el trabajo es importante en la vida de las personas, ya que ocupan mucho de su tiempo en él y es una fuente de estabilidad financiera, pero también es una fuente de gran estrés. Por lo tanto, el trabajo debe ser atractivo y debe dar a las personas un cierto grado de satisfacción. sobre el desempeño alcanzado y propiciar actividades de capacitación constantes para los docentes, además de proveer soporte y guía para la organización y planificación de la carga laboral docente. En cuanto a los sostenedores de los establecimientos educacionales, deberían considerar la formación de los directores en habilidades de liderazgo. En este sentido, se ha integrar una práctica de liderazgo que incluya elementos del medio y valores institucionales, la participación de los colaboradores y la comunicación oportuna, junto con una clara motivación de aquella persona encargada de liderar un establecimiento educacional, quien propenderá hacia la eficiencia de los procesos de enseñanza- aprendizaje e impactará en la satisfacción, clima organizacional y desempeño del cuerpo docente.

En segundo lugar, en el presente estudio, los mayores indicadores de desequilibrio entre la vida y trabajo se atribuyen a la falta de tiempo para la vida personal; cantidad de trabajo; estrés; prisas y agobios y carga de responsabilidad. La excesiva carga laboral se denota indicando falta de tiempo para desarrollar su vida personal, estrés, baja remuneración e imposibilidad de desconectarse de sus labores al llegar al hogar. 
Tabla 3. Distribución de puntuaciones por ítem

\begin{tabular}{|c|c|c|c|}
\hline & $\mathbf{n}$ & Media & Desv. típ. \\
\hline i1 Interrupciones molestas en el trabajo & 380 & 6,20 & 2,540 \\
\hline i2 Consecuencias negativas para la salud en el trabajo & 380 & 6,83 & 2,568 \\
\hline i3 Falta de tiempo para la vida personal. & 380 & 8,46 & 2,173 \\
\hline i4 Incomodidad física en el trabajo. & 380 & 5,70 & 2,486 \\
\hline i5 Conflictos con otras personas en el trabajo. & 380 & 4,15 & 2,366 \\
\hline i6 Variedad en mi trabajo & 380 & 5,18 & 2,500 \\
\hline i7 Posibilidad de expresar lo que pienso y necesito & 380 & 4,65 & 2,614 \\
\hline i8 Apoyo de mis jefes & 380 & 5,13 & 2,533 \\
\hline i9 Posibilidades de que mis propuestas sean escuchadas y aplicadas & 380 & 4,80 & 2,420 \\
\hline i10 Satisfacción con el sueldo. & 380 & 2,87 & 2,153 \\
\hline i11 Posibilidad de ser creativo & 380 & 5,64 & 2,624 \\
\hline i12 Mi establecimiento trata de mejorar mi calidad de vida & 380 & 3,41 & 2,299 \\
\hline i13 Reconocimiento de mi esfuerzo en el trabajo & 380 & 3,93 & 2,544 \\
\hline i14 Recibo información de los resultados de mi trabajo & 380 & 4,41 & 2,568 \\
\hline i15 Posibilidad de promoción en mi trabajo & 380 & 2,86 & 2,158 \\
\hline i16 Cantidad de trabajo & 380 & 8,57 & 2,035 \\
\hline ¡17 Estrés & 380 & 8,30 & 2,038 \\
\hline i18 Prisas y agobios & 380 & 8,17 & 2,143 \\
\hline i19 Presión recibida para realizar mi trabajo & 380 & 7,55 & 2,372 \\
\hline i20 Presión recibida para mantener la calidad del trabajo & 380 & 7,76 & 2,379 \\
\hline i21 Mi trabajo es importante para la vida de otras personas & 380 & 8,51 & 2,229 \\
\hline i22 Carga de responsabilidad & 380 & 8,61 & 1,841 \\
\hline i23 Lo que tengo que hacer queda claro & 380 & 6,76 & 2,312 \\
\hline i24 Autonomía o libertad de decisión & 380 & 4,78 & 2,309 \\
\hline i25 Apoyo de mi familia & 380 & 7,99 & 2,516 \\
\hline i26 Apoyo de mis compañeros & 380 & 6,49 & 2,382 \\
\hline i27 Apoyo de mis subordinados & 380 & 5,40 & 2,608 \\
\hline i28 Ganas de ser creativo & 380 & 7,92 & 2,382 \\
\hline i29 Orgullo del trabajo & 380 & 7,67 & 2,561 \\
\hline i30 Motivación & 380 & 6,43 & 2,801 \\
\hline ¡31 Satisfacción con el trabajo & 380 & 5,82 & 2,522 \\
\hline i32 Estoy capacitado para realizar mi trabajo & 380 & 8,58 & 1,750 \\
\hline i33 Se exige capacitación para realizar mi trabajo & 380 & 6,59 & 3,072 \\
\hline i34 Me desconecto al acabar la jornada de trabajo & 380 & 2,71 & 2,522 \\
\hline
\end{tabular}

Con respecto a las demandas laborales, estas se relacionan con la sobrecarga de trabajo, los problemas de conducta de los alumnos, el equipo deficiente, el clima organizacional, los conflictos interpersonales y los problemas de los estudiantes. La mencionada sobrecarga laboral viene dada por las bajas rentas que perciben los docentes, lo cual los lleva a buscar trabajos alternativos, incrementando su horario de trabajo, reduciendo sus tiempos de descanso y recreación y, por consiguiente, disminuyendo su calidad de vida, llevándoles a un desgaste físico y mental (Andrade et al., 2003 como se citó en Sánchez \& Corte, 2012; Domich \& Faivovich, 1994; Esteve, 2006; Parra, 2005; Robalino 2005; Rodríguez, 2012; Tenti, 2005; Vaillant, 2004).

En relación con lo anterior, en Estados Unidos existe evidencia que relaciona el abandono de la profesión docente con los bajos salarios, aunque también influyen en esta decisión otros factores, entre los cuales 
Tabla 4. Diferencias entre género femenino y género masculino

\begin{tabular}{llccc}
\hline & Género & $\mathrm{n}$ & Rango promedio & Sig. asintót. (bilateral) \\
\hline \multirow{2}{*}{ Factor I: Apoyo directivo } & Femenino & 308 & 190,81 & 0,908 \\
& Masculino & 72 & 189,15 & 0,358 \\
\hline \multirow{2}{*}{ Factor II: Carga de trabajo } & Femenino & 308 & 188,00 & $0,011\left(^{*}\right)$ \\
& Masculino & 72 & 201,22 & 183,56 \\
\hline \multirow{2}{*}{ Factor III: Motivación intrínseca } & Femenino & 308 & 220,17 & 0,109 \\
\hline \multirow{2}{*}{ Calidad de vida profesional } & Masculino & 72 & 186,14 & 209,15 \\
\hline
\end{tabular}

$\left(^{*}\right) \cup$ de Mann-Whitney. $p \leq 0,05 . n=380$.

sobresalen el escaso soporte directivo-administrativo, los problemas disciplinarios de los estudiantes y la ausencia de influencia en las decisiones sustantivas sobre el aula y el establecimiento. En Chile, los profesores perciben menores rentas con respecto a sus pares, encontrándose que los titulados de las carreras de educación representan un $50 \%$ del ingreso laboral promedio comparado con el de otras carreras profesionales, tanto al segundo como al cuarto año de titularse

En tercer lugar y asociado al tipo de dependencia del establecimiento en el cual se desempeñan, los profesores que se trabajan en una institución dependiente de un municipio experimentan un mayor apoyo por parte de sus superiores en el desempeño de sus funciones. De este modo, el director del establecimiento y su equipo directivo siguen siendo el foco de atención en relación al papel vital que juegan en el desempeño exitoso de la organización. Las tendencias recientes hacia la descentralización en la toma de decisiones señalan que un buen director debe ser a la vez un educador profesional, un líder y un gerente exitoso. En este (Valenzuela, Sevilla, Bellei \& de los Ríos, 2010).

sentido, debe demostrar su liderazgo en el establecimiento e inspirar a todos los interesados a colaborar activamente en la consecución de la misión educativa; las metas a corto y largo plazo; la instrucción y el cumplimiento de altos estándares para los estudiantes, el personal y los procesos organizativos (Friedman, 2002).

En la misma línea, los estudios realizados por Castillo, González y Puga (2011) acerca de la gestión en establecimientos municipales y particulares subvencionados evidenciaron que en los establecimientos municipales se tiende a enfatizar la formación ciudadana; en cambio, los establecimientos particulares subvencionados privilegian una formación valórica o la formación de hábitos, además de centrarse en la obtención de resultados orientados a la excelencia académica. Por otra parte, en las organizaciones de régimen particular subvencionado se encuentran estilos de dirección autoritarios, en donde la participación en la toma de decisiones y comunicación es menor. En lo relativo a la estabilidad, los docentes de centros educativos particulares subvencionados tienen en promedio ocho años de antigüedad en su actual trabajo, mientras que Tabla 5. Diferencias según tipo de dependencia

\begin{tabular}{|c|c|c|c|c|}
\hline & Tipo de dependencia & $\mathrm{n}$ & Rango promedio & Sig. asintót. (bilateral) \\
\hline & Municipal & 121 & 203,71 & \\
\hline \multirow[t]{3}{*}{ Factor I: Apoyo directivo } & Particular Subvencionado & 234 & 189,04 & $0,030(*)$ \\
\hline & Particular Pagado & 25 & 140,20 & \\
\hline & Municipal & 121 & 210,17 & \\
\hline \multirow[t]{3}{*}{ Factor II: Carga de trabajo } & Particular Subvencionado & 234 & 185,11 & $0,014(*)$ \\
\hline & Particular Pagado & 25 & 145,72 & \\
\hline & Municipal & 121 & 185,90 & \\
\hline \multirow[t]{3}{*}{ Factor III: Motivación intrínseca } & Particular Subvencionado & 234 & 190,70 & 0,583 \\
\hline & Particular Pagado & 25 & 210,92 & \\
\hline & Municipal & 121 & 200,33 & \\
\hline \multirow[t]{2}{*}{ Calidad de vida profesional } & Particular Subvencionado & 234 & 187,78 & 0,345 \\
\hline & Particular Pagado & 25 & 168,34 & \\
\hline
\end{tabular}

$(*)$ H de Kruskal-Wallis. $p \leq 0,05 . n=380$. 
Tabla 6. Diferencias en calidad de vida entre docentes de la Región Metropolitana y el resto del país.

\begin{tabular}{|c|c|c|c|c|}
\hline & Región & $\mathrm{n}$ & Rango promedio & Sig. asintót. (bilateral) \\
\hline \multirow{2}{*}{ Factor I: Apoyo directivo } & Regiones & 144 & 189,09 & \multirow{2}{*}{0,845} \\
\hline & RM Región Metropolitana & 236 & 191,36 & \\
\hline \multirow{2}{*}{ Factor II: Carga de trabajo } & Regiones & 144 & 172,82 & \multirow{2}{*}{$0,014\left(^{*}\right)$} \\
\hline & RM Región Metropolitana & 236 & 201,29 & \\
\hline \multirow{2}{*}{ Factor III: Motivación intrínseca } & Regiones & 144 & 171,78 & \multirow{2}{*}{$0,009\left(^{*}\right)$} \\
\hline & RM Región Metropolitana & 236 & 201,92 & \\
\hline \multirow{2}{*}{ Calidad de vida profesional } & Regiones & 144 & 174,18 & \multirow{2}{*}{$0,024\left(^{*}\right)$} \\
\hline & RM Región Metropolitana & 236 & 200,46 & \\
\hline
\end{tabular}

(*) $\cup$ de Mann-Whitney. $p \leq 0,05 . n=380$.

en los profesores de establecimientos municipales, este indicador sube significativamente a diez años (Corvalán, Elacqua \& Salazar, 2009). Estas características demuestran y ponen de manifiesto la precariedad de las condiciones de trabajo en las cuales se desempeñan los maestros que se desempeñan en los llamados establecimientos con fines de lucro, afectando a su calidad de vida laboral.

En conclusión, urge establecer una línea estratégica de gestión directiva que busque fortalecer y dinamizar el manejo de recursos humanos y materiales, las relaciones humanas, los procesos de toma de decisión y las formas organizativas. Hasta el momento, los esfuerzos se han centrado fundamentalmente en lo academicista, impulsándose procesos de capacitación de los directivos a través del acceso a estudios de diplomado o magíster en temas de administración educacional. Sin embargo, las autoridades no han optado por una vinculación clara o esfuerzo orientados al trabajo en equipo, la planificación estratégica, el diseño y ejecución de proyectos educativos y el liderazgo compartido que apunten a favorecer, en los establecimientos educacionales, un clima de colaboración, intercambio de experiencias y desarrollo de los talentos de cada uno de sus miembros.

\section{Referencias}

Arellano, J. (2005). Prioridades estratégicas para mejorar la calidad de la educación en Chile. Serie Estudios Socio/Económicos, 25(28), 1-24. Recuperado de http:// www.cieplan.cl/media/publicaciones/archivos/48/ Documento Completo.pdf

Brunner, J. (2003). Informe capital humano en Chile. Santiago, Chile: Universidad Adolfo lbáñez.

Cabezas, C. (1998). Síndrome de desgaste profesional, estrés laboral y calidad de vida profesional. Formación Médica Continua, 5(8), 491-492. Recuperado de http:// www.fmc.es/es/sindrome-desgaste-profesionalestreslaboral/articulo/4766/

Caboblanco, M., Martín, J., Páez, M., Garijo, J., Rodríguez, A. \& Cortés, J. (2004). Características métricas del Cues- tionario de Calidad de Vida Profesional (CVP-35). Gaceta Sanitaria, 18(2), 129-136. doi:10.1016/S02139111(04)71817-8

Cañón, S. \& Martínez, G. (2011). Factores laborales psicosociales y calidad de vida laboral de los trabajadores de la salud de ASSBASALUD E.S.E Manizales (Colombia). Archivos de Medicina, 11(2), 114-126. Recuperado de http://www.redalyc.org/pdf/2738/273821489004. pdf

Casas, J., Lorenzo, S. \& Cañas, J. (2002). Dimensiones y medición de calidad de vida laboral en profesionales sanitarios. Revista de Administración Sanitaria, 6(23), 143160. Recuperado de https://rrhh-esgc.wikispaces. com/file/view/Calidad+de+vida+laboral.pdf

Castillo, P., González, A. \& Puga, I. (2011). Gestión y efectividad en educación: evidencias comparativas entre establecimientos municipales y particulares subvencionados. Revista Estudios Pedagógicos, 37(1), 187-206. doi:10.4067/S0718-07052011000100010

Cegarra-Leiva, D., Sánchez-Vidal, M. \& Cegarra-Navarro, J. (2012). Understanding the link between work life balance practices and organisational outcomes in SMEs. The mediating effect of a supportive culture. Personnel Review, 41(3), 359-379. doi:10.1108/00483481211212986

Cornejo, R. (2009). Condiciones de trabajo y bienestar/malestar docente en profesores de enseñanza media de Santiago de Chile. Educação \& Sociedade, 30(107), 409-426. doi:10.1590/S0101-73302009000200006

Corvalán, J., Elacqua, G. \& Salazar, F. (2009). El sector particular subvencionado en Chile. Tipologización y perspectivas frente a las nuevas regulaciones (FONIDE N69). Santiago, Chile: Centro de Investigación y Desarrollo de la Educación (CIDE) y Centro de Políticas Comparadas de Educación (CPCE).

Corvalán, O. (2011). Cambios en la gestión educativa y rol de los liceos municipalizados. Revista electrónica de Desarrollo de Competencias (REDEC), 1(7), 198-215. Recuperado de http://redec.utalca.cl/index.php/redec/ article/view/64

Dirección del Trabajo. (2011). Encuesta Laboral (ENCLA) 2011. Recuperado de http://www.dt.gob.cl/documentacion/1612/w3-article-101347.html 
Domich, C. \& Faivovich, M. (1994). Diagnóstico de la salud mental en profesores de ocho escuelas básicas municipalizadas de la comuna de Santiago. Revista Chilena de Psicología, 15(2), 15-21.

Dueñas, G. \& Giolito, E. (2012). Separación y divorcio: impacto sobre familias en Chile. Observatorio Económico, 66. Recuperado de http://fen.uahurtado.cl/2012/articulos/observatorio-economico/separacion-y-divorcio-impacto-sobre-familias-en-chile/

Esteve, J. (2006). Identidad y desafíos de la condición docente. El Oficio de Docente. Vocación, Trabajo y Profesión en el Siglo XXI. Buenos Aires: Siglo Veintiuno Editores.

Facultad de Economía y Negocios, USS. (2014). Cuarto Informe de Deuda Personal Deudores Morosos a Marzo 2014. Recuperado de http://www.uss.cl/newsletter-uss/wp-content/uploads/sites/19/2015/03/IV-Informe-de-Deuda-Persona-USS-Equifax.pdf

Fernet, C., Guay, F., Senécal, C. \& Austin, S. (2012). Predicting intraindividual changes in teacher burnout: The role of perceived school environment and motivational factors. Teaching and Teacher Education, 28, 514-525. doi:10.1016/j.tate.2011.11.013

Friedman, I. (2002). Burnout in school principals: role related antecedents. Social Psychology of Education, 5, 229251. doi:10.1023/A:1016321210858

Fundación SOL. (2013). Panorama General de los Trabajadores Dependientes que ganan el salario mínimo (SM) usando la Encuesta Casen 2011. Recuperado de http:// www.fundacionsol.cl/wp-content/uploads/2015/06/ Panorama-Salario-Mi\%CC\%81nimo-2013-Final.pdf

Garrido, P., Ansoleaga, E., Tomicic, A., Domínguez, C., CastiIlo, S., Lucero, C. \& Martínez, C. (2013). Afecciones de Salud Mental y el Proceso de Retorno al Trabajo: Una Revisión Sistemática. Ciencia \& Trabajo, 15(48), 105113. doi:10.4067/S0718-24492013000300002

Genesi, M. \& Suárez, F. (2010). Management of quality of the human talent in the intelligent educative organizations. Revista Científica Electrónica de Ciencias Humanas, 17(6) 116-155. Recuperado de https://dialnet. unirioja.es/descarga/articulo/3632926.pdf

González, R., Hidalgo, G. \& Salazar, J. (2006). Calidad de vida en el trabajo: un término de moda con problemas de conceptuación. Psicología y Salud, 17(1), 115-123. Recuperado de http://revistas.uv.mx/index.php/psicysalud/article/view/746

Goswami, M. (2013). A Study of Burnout of Secondary School Teachers in Relation to their Job Satisfaction. Journal Of Humanities And Social Science, 10(1), 18-26. Recuperado de http://iosrjournals.org/iosr-jhss/papers/ Vol10-issue1/C01011826.pdf?id=6194

Guerrero, E. \& Rubio, J. (2008). Fuentes de estrés y Síndrome de «Burnout» en orientadores de institutos de Enseñanza Secundaria. Revista de Educación, 347, 229-254. Recuperado de http://www.mecd.gob.es/ revista-de-educacion/numeros-revista-educacion/ numeros-anteriores/2008/re347/re347 11.htm

Guic, E., Bilbao, A. \& Bertin, C. (2002). Estrés laboral y salud en una muestra de ejecutivos chilenos. Revista Médica de Chile, 130(10), 1101-1112. doi:10.4067/S003498872002001000004

Hernández, R., Fernández, C. \& Baptista, P. (2010). Metodología de la investigación (5a. ed.). México: Mc. Graw Hill.

Karasek, R. (1989). The Political Implications of Psychosocial Work Redesign: A Model of the Psychosocial Class Structure. International Journal of Health Service, 19(3), 481-508. doi:10.2190/66AM-Q4PF-PUHK-5BT1

López, P. \& Gallegos, V. (2014). Prácticas de liderazgo y el rol mediador de la eficacia colectiva en la satisfacción laboral de los docentes. Estudios Pedagógicos, 40(1), 163-178. doi:10.4067/S0718-07052014000100010

Malik, K. (2013). Informe sobre Desarrollo Humano 2013. El ascenso del Sur: Progreso humano en un mundo diverso. Canadá: Gilmore Printing Services Inc. Recuperado de http://www.undp.org/content/dam/venezuela/ docs/undp ve IDH 2013.pdf

Martín, J., Cortés, A., Morente, M., Caboblanco, M., Garijo, J. \& Rodríguez, A. (2004). Características métricas del Cuestionario de Calidad de Vida Profesional (CVP35). Gaceta Sanitaria, 18(2), 129-36. doi:10.1016/ S0213-9111(04)71817-8

Maureira, O. (2006). Dirección y Eficacia Escolar, una relación fundamental. Revista Electrónica Iberoamericana sobre Calidad, Eficacia y Cambio en Educación (REICE), 4(4), 1-10. Recuperado de http://www.rinace.net/arts/ vol4num4e/art1.htm

Maxfield, S. (2005). Modifying best practices in women's advancement for the Latin American context. Women in Management Review, 20(4), 249-261. doi:10.1108/09649420510599070

Ministerio de Educación. (1999). Docencia: una de las funciones más exigidas. Revista de Educación, 262, 23-25.

Nava, M. (2012). La calidad de vida: Análisis multidimensional. Revista Enfermería Neurológica, 11(3), 129-137. Recuperado de http://new.medigraphic.com/cgi-bin/resumen.cgi?IDARTICULO=38334

Ollier-Malaterre, A., Valcour, M., Den Dulk, L. \& Kossek, E. (2013). Theorizing national context to develop comparative work-life research: A review and research agenda. European Management Journal, 31, 433-447. doi:10.1016/j.emj.2013.05.002

Parra, M. (2006). Análisis comparativo. En M. Robalino \& A. Körner (Coords.), Condiciones de trabajo y salud docente (pp. 19-40). Santiago, Chile: UNESCO.

Ponce, C. (2002). Burnout y estrategias de afrontamiento en profesores de enseñanza básica. Revista Psykhé, 11(2), 71-88. Recuperado de http://www.psykhe.cl/index.php/psykhe/article/view/429

Programa de las Naciones Unidas para el Desarrollo. (2012). Desarrollo humano en Chile 2012. Bienestar subjetivo: el desafío de repensar el desarrollo. Recuperado de http://www.cl.undp.org/content/chile/es/home/library/human development/publication 3.htm

Programa de las Naciones Unidas para el Desarrollo. (2014). 
Chile se ubica en el primer lugar en Desarrollo Humano entre los países de América Latina y el Caribe. Recuperado de http://www.cl.undp.org/content/chile/ es/home/presscenter/pressreleases/2014/07/24/ chile-se-ubica-en-el-primer-lugar-en-desarrollo-humano-entre-los-pa-ses-de-am-rica-latina-y-el-caribe.html

Quezada, F., Sanhueza, A. \& Silva, F. (2010). Diagnóstico de la calidad de vida laboral percibida por los trabajadores de cuatro servicios clínicos del Complejo Asistencial "Dr. Víctor Ríos Ruiz" de Los Ángeles (CAVRR). Horizontes Empresariales, 9(1), 55-68. Recuperado de http://www.ubiobio.cl/miweb/webfile/media/42/ version\%209-1/diagnostico.pdf

Redondo, J. (2009). La educación chilena en una encrucijada histórica. Revista Diversia, 1(1), 13-39. Recuperado de http://www.cidpa.cl/wp-content/uploads/2013/05/ Revista-Diversia-01.pdf

Robalino, M. (2005). Las condiciones de trabajo y salud docente: aportes, alcances y límites del estudio. En M. Robalino \& A. Körner (Coords.), Condiciones de trabajo y salud docente (pp. 11-18). Santiago, Chile: UNESCO.

Rodríguez, L. (2012). Condiciones de trabajo docente: Aportes de México en un estudio latinoamericano. Revista Diálogos Educativos, 12(24), 18-27. Recuperado de http://revistas.umce.cl/dialogoseducativos/article/ viewFile/81/90

Romanik, K. (2011). Informe de Actualidad Laboral No2: El mobbing y su tratamiento en la legislación laboral (Informe de Actualidad Laboral 2). Recuperado de http://www. dt.gob.cl/documentacion/1612/articles-100418 recurso 1.pdf

Romeo, M., Yepes-Baldó, M. \& Berger, R. (2014). Contribución española e iberoamericana al estudio del "work life balance": claves para la intervención. Papeles del Psicólogo, 35(1), 48-58. Recuperado de http://www. papelesdelpsicologo.es/pdf/2321.pdf

Rueda, S. (1996). Habitabilidad y calidad de vida. Cuadernos de Investigación Urbanística, 42, 29-33. Recuperado de http://habitat.aq.upm.es/select-sost/ac3.html

Sánchez, M. \& Corte, F. (2012). La precarización del trabajo. El caso de los maestros de educación básica en América Latina. Revista Latinoamericana de Estudios Educativos, 42(1), 25-54. Recuperado de http://www. redalyc.org/pdf/270/27023323003.pdf

Sánchez, D., March y Cerda, M. \& Ballester, L. (2015). Malestar social y malestar docente: Una investigación sobre el síndrome de desgaste profesional burnout y su incidencia socioeducativa. Aula, 21, 245-257. doi:10.14201/ aula201521245257

Segurado, A. \& Agulló, E. (2002). Calidad de vida laboral: hacia un enfoque integrador desde la psicología social.
Psicothema, 12(4), 828-836. Recuperado de http:// www.psicothema.com/psicothema.asp?id=806

Tenti, E. (2005). La condición docente. Análisis comparado de la Argentina, Brasil, Perú y Uruguay. Buenos Aires: Siglo XXI.

Uribe, M. (2005). El liderazgo docente en la construcción de la cultura escolar de calidad: un desafío de orden superior. Revista PRELAC-UNESCO, 1-11. Recuperado de http://www.ceppe.cl/images/stories/recursos/publicaciones/Mario\%20Uribe/El-liderazgo-docente.pdf

Vaillant, D. (2004). Construcción de la Profesión Docente en América Latina. Tendencias, temas y debates, GTDPREAL (Documento de Trabajo 31). Recuperado de http://www.oei.es/historico/docentes/articulos/ construccion profesion docente AL vaillant.pdf

Verdugo, J., Guzmán, J., Moy, N., Meda, R. \& González, O. (2008). Factores que influyen en la calidad de vida de profesores universitarios. Psicología y Salud, 18(1), 2736. Recuperado de http://revistas.uv.mx/index.php/ psicysalud/article/view/672

Villarreal, E. (2005). La efectividad de la gestión escolar depende de la formación del recurso humano como factor, actor y promotor del cambio dentro de los procesos, dimensiones y políticas educativas. Revista Iberoamericana de Educación, 37(2), 1-4. Recuperado de http://rieoei.org/1083.htm

Walton, R. (1973). Quality of Working Life: What is it? Sloan Management Review, 15(1), 11-21.

Weinstein, J. (2002). Calidad y Gestión en Educación: Condiciones y desafíos. Pensamiento Educativo, 31, 50-71. Recuperado de http://pensamientoeducativo.uc.cl/ index.php/pel/article/view/229

Zinser, O. (1987). Psicología Experimental. México: McGraw Hill. 